\title{
Controlling a system for underwater visual inspection
}

\section{Paulo L. J. Drews-Jr. ${ }^{1}$, Vinícius N. Kuhn² and Sebastião C. P. Gomes ${ }^{3}$}

1 C3/FURG, Brazil, Email: paulodrews@furg.br

2 C. Charqueadas/IFSUL, Brazil, Email: vinicius@charqueadas.ifsul.edu.br

3 IMEF/FURG, Brazil, Email: sebastiaogomes@furg.br

\begin{abstract}
Nowadays, the ocean plays a fundamental role in the global economy, mainly due to oil extraction industry. It makes the environment be populated with human-made structures that needs to be inspected and maintained. In this context, this paper details a system for online detect an underwater cable-like target using computer vision algorithms, as well as an automatic control of a vehicle to tracking it. This system could be used to assist a human operator during visual inspection tasks. This work is concluded with a series of tests and analyses aiming to the system validation.
\end{abstract}

\section{Keywords}

Control, computer vision, tracking

\section{Nomenclature}

e error signal sampled

$f \quad$ focal distance

Z distance between target and the camera

$k \quad$ distance between the lens and the focal point

$\mathrm{Ki}$ integral gain

$K d \quad$ derivative gain

$K p \quad$ proportional gain

$N \quad$ number of elements in the forgetting window $u \quad$ control action

Width width of the target

\section{Subscripts}

$p x \quad$ relative to the image pixels

$s \quad$ relative to the surge motion controller

tgt relative to the target

1 relative to the first parameter of the camera

2 relative to the second parameter of the camera 


\section{Introduction}

The ocean covers more than $70 \%$ of the planet's surface. The study of this environment becomes very important in different areas, mainly in oil extraction industry. Nevertheless, much of the underwater environment is still unknown to the man, either by size or by adverse environmental conditions. In these situations, unmanned underwater vehicles become an important tool because they allow, without risking human lives, to perform inspections, collect data, perform construction work, and installation of underwater structures, etc.

The inspection of underwater installations, such as power cables, telecommunications, and pipelines, is performed by trained professionals who, from the surface, controls a ROV (Remotely Operated Vehicle) and inspect these structures based on images captured by an embedded video camera (Drews Jr. et al., 2008). Controlling a ROV is a hard, slow, tiring, and error-prone task because it requires experience and constant operator attention to a console in order to maneuver the vehicle (Gomes et al., 2005). Moreover, the task complexity is increased by the ocean currents action and by lack of underwater images quality. Therefore, the automation of this process can constitute an important improvement with respect to errors, working time, and cost.

However, underwater vehicles are also characterized by having a difficult instrumentation, i.e., accurately determine the pose of the vehicle from inertial sensors is a complicated task and can be very expensive. In this context the use of images could help in inspection task, because it provides information to the ROV's control system. In this situation, the human operator only needs to pay attention to the image in order to inspect the structure (Drews et al., 2013).

Some works were proposed in the topic of cable-pipeline tracking. In the work of Wirth et al. (2007), an automatic method to identify cable and pipelines is proposed. This work is based on particle filter to estimate the pose of cable-pipeline structures. Although the impressive results obtained the temporal performance and computational power required are not compatible with the present work. Narimani et al. (2009) proposed a vision based system to tracking cable and pipes. The focus of this work is the development of image processing algorithms to improve a nonlinear control using sliding mode. Different from the present work, the control technique performance is the main purpose.

This work proposes a tracking algorithm based on computer vision techniques in order to help a human operator to accomplished inspection tasks. We demonstrate the feasibility of the proposed algorithm controlling a distance between a target and a ROV.

This paper is an extension of the previous paper Drews Jr. et al. (2012), where the tracking system was initially proposed.
In the present paper, the method is better explained, as well as a surge controlling system is presented and tested. Differently from Kuhn et al. (2013), where the focus is in the control of the ROV in many degree of freedom, the present work is focused in the image processing method and the surge control.

\section{Tracking system}

This work proposed an efficient method to detect cablepipeline structure in underwater environment. The structure is called as target in this work. Fig. 1 presents an overview of the proposed method. The image changes throughout the processing are presented in Fig. 2.

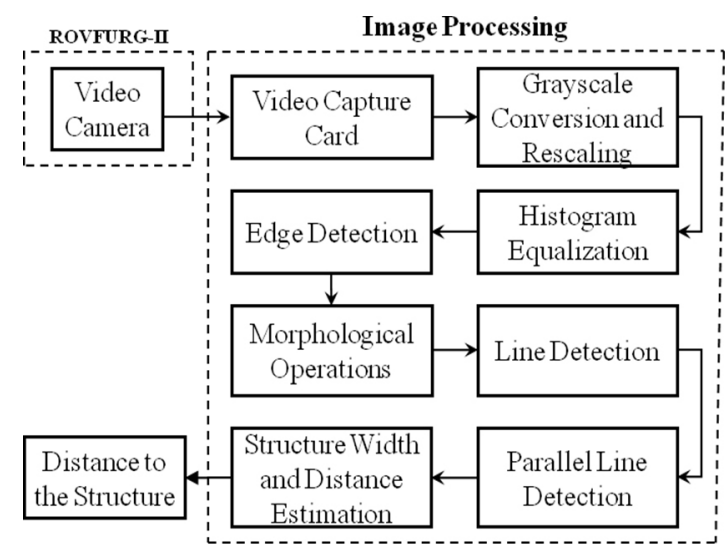

Fig. 1 Image Processing Methodology.

Initially, the camera images are received by a video capture card. Once the image is available, it is resized to $320 \times 240$ pixels using bilinear interpolation. Then, the resized image is converted to grayscale, losing its color characteristics, since this work does not make any assumption about the object color to be tracked, just about its shape. These two initial procedures reduce significantly the computational cost and are essential in order to improve the system performance. Fig. 2-(a) shows the resized image, and in Fig. 2-(b) shows the resized image after grayscale conversion.

The next step is to apply an edge detector. In this case, the Canny algorithm (Canny, 1986) is applied to the grayscale images. This operator produces as output an image with the positions of the detected intensity discontinuities. This processing result is shown in Fig. 2-(c).

To assist the next process, detection of lines, two morphological operators are applied: a dilation and erosion. 
They highlight the edges found by Canny Detector. Fig. 2 -(d) presents the resulting image of these operators.

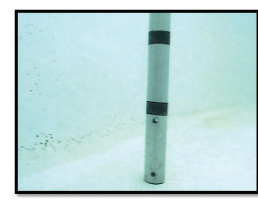

(a)

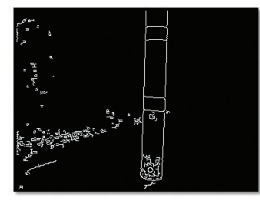

(c)

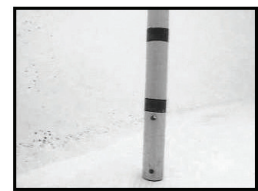

(b)

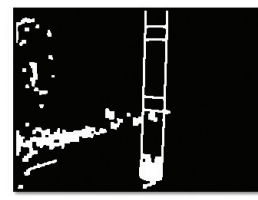

(d)

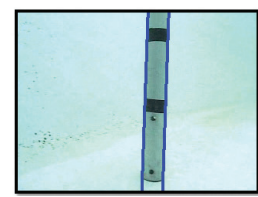

(e)

Fig. 2 Example of the images obtained in each step using the proposed method. (a) shows the original image, (b) after grayscale conversion, (c) after edge detection using Canny algorithm, (d) after morphological operators, (d) after detection of parallel lines.

After the highlight by the morphological operators, the Hough transform (Duda and Hart, 1972) is used as operator to detect lines. In this process, pixels are converted from Euclidean space to line space, and then the intersection points between them are found and grouped into line segments. From this information, the longest line segments that are parallel among themselves are searched (target contours) and two parallel lines along them are drawn. These two lines can be seen in Fig. 2-(e).

The distance between these two parallel lines corresponds to the external diameter of the target. The method used to compute this distance is detailed in the next section. In case of failure in the target identification, the distance measurement is not updated, remaining with the last valid value identified. It considers that our method is able to detect soon again the target.

The method proposed is able to detect the target in different rotation, scale and displacement condition using a small processing time. The system is able to trackin in $60 \mathrm{~Hz}$ with few fails during the detection, as we show in Sec. 3. Other approaches as Kalman Filter (Kalman, 1960) and Particle Filter (Doucet et al., 2000) could be applied to improve the estimation, but due the restriction in computational time; the small improved in the results; and the slow dynamic of the system, they are not used in this work.

The distance between these two parallel lines corresponds to the external diameter of the metallic structure pipe type. Thus, the distance from the structure to the vehicle can be obtained from Eq. (2).

In case of failure in structure identification, the distance measurement is not updated, remaining with the last valid value identified. This algorithm does not foresee situations in which the structure is out of sight of the camera: in these cases, the system control must be changed to the manual mode.

\subsection{Calibration process}

The goal of this tracking system is determine the distance to the target. In this way, computer vision techniques are applied to images captured by the camera, in order to identify know patterns, as shown in previous section. Once the algorithm has identified the target, the distance from the ROV to it can be estimated based on their size in the captured image (number of pixels) and its actual size (previously known).

From the pinhole camera model (Szelinski, 2010), one obtains:

Width $_{t g t} \cdot f=$ Width $_{p x} \cdot(Z-k)$

where: Width $_{t g t}$ is the object width in centimeters, Width is the object width in the image in pixels, $Z$ is the actual distance from the object to the camera in meters, and $k$ is an unknown constant which defines the distance between the camera outside and the optical center, and $f$ is the focal length in centimeters. This last information is previously known through the calibration process as proposed in Zhang (1999). Eq. 1 uses as prior knowledge the focal distance value $(f)$, object width in the image (Widthpx), and $k$. Thus, it making possible to establish the real distance from the object to the camera.

As $k$ is initially unknown, we determine the constants values and a more accurate focal length value by a specific experiment, since they are critical parameters to obtain the actual distance from the camera to the object. In this experiment, it was used a pipe-like metallic target with 3.2 $\mathrm{cm}$ external diameter, presented in Fig. 3. The tests were performed with the submersed ROV for the light effects in water to be considered.

Table 1 Experimental data acquired using the ROV and the target

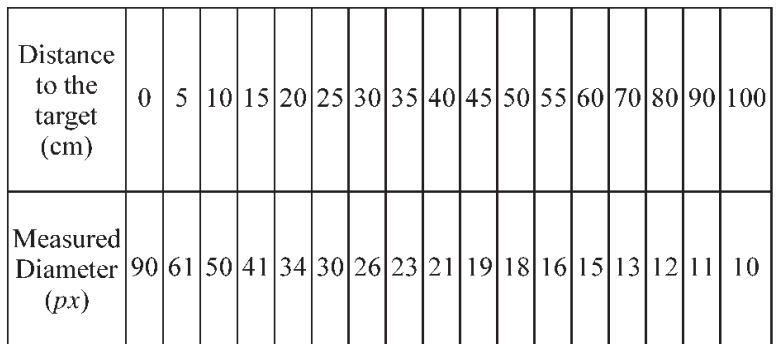




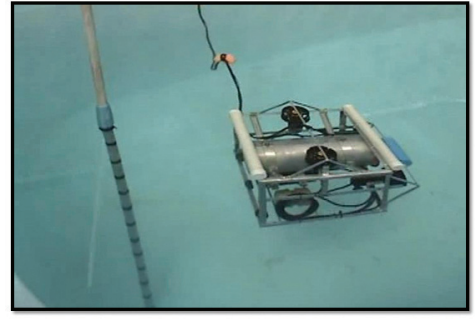

(a)

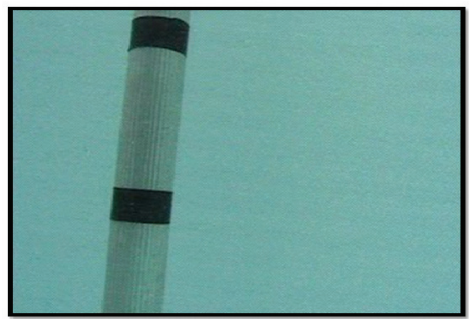

(b)

Fig. 3 Pipe-like metallic target and the ROV. (a) The external image of the testbed, and (b) the image internally acquired by the ROV of the target with $32 \mathrm{~mm}$ of diameter.

Initially, this structure was placed close to the video camera, perpendicular, and its image was captured. In the sequence, the structure was displaced at a distance of $5 \mathrm{~cm}$ from the camera and the image was captured again. This procedure was accomplished and the results are shown in Table 1. Fig. 3 -(b) shows the example for the distance of $15 \mathrm{~cm}$, where the diameter in pixel was obtained as 41 .

From Eq. 1, one obtains:

$Z=k_{1}+k_{2} /$ Width $_{p x}$

where: $k_{1}$ is the constant $k$ and $\mathrm{k}_{2}$ is $f$. Width $h_{\text {tgt }}$, both in Eq. 1 .

By using experimental data, and Eq. 2, which relates the real distance $(Z)$ with the width measured in pixels $\left(\right.$ Width $\left._{p x}\right)$, we applied the least squares method to find the values of the constants $k_{I}$ and $k_{2}$. The obtained values were: $k_{1}=-11.90$ $\mathrm{cm}$ and $k_{2}=1083.29 \mathrm{~cm} . p x$ (where px is number of pixels). The negative value of $k_{l}$ is related with the referential defined to the Eq. 2.

Taking into account the analytical estimation of parameters: $f=494.17 \mathrm{~cm}$ with standard deviation of \pm 158.18 (in $p x$ ) and $W_{i d t h} h_{t g t}=3.2 \mathrm{~cm}$, by the Eq. 1. The analytical value of $k_{1}$ is not available by the manufactor, and we are not able to estimate it. However, $k_{2}$ results in 1581.35 cm.px with values within the expected range: from 1075.17 to 2087.52 cm.px. The difference from $k_{2}$ estimated analytically to the experimental one, is due to high radial distortion presented in the camera calibration process to estimate the focal distance. Moreover, the real width estimation shows small variations that are difficult to distinguish and can affect the determination of this constant.
Fig. 4 shows the experimental response curve and the response curve obtained by Eq. 2 . It can be observed that the curve of Eq. 2 approaches considerably the experimental one and, for this reason, it has been adopted for the online computation of the distance from the image captured by the camera. The comparative between experimental and analytical distance is shown in Fig.4-(a), and the error obtained between this two curves is shown in Fig. 4-(b).

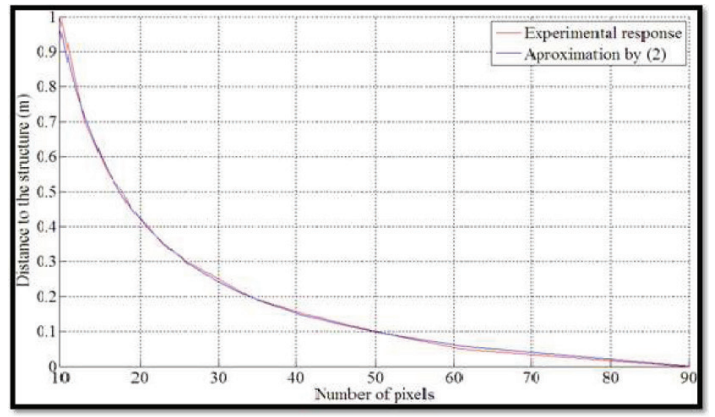

(a)

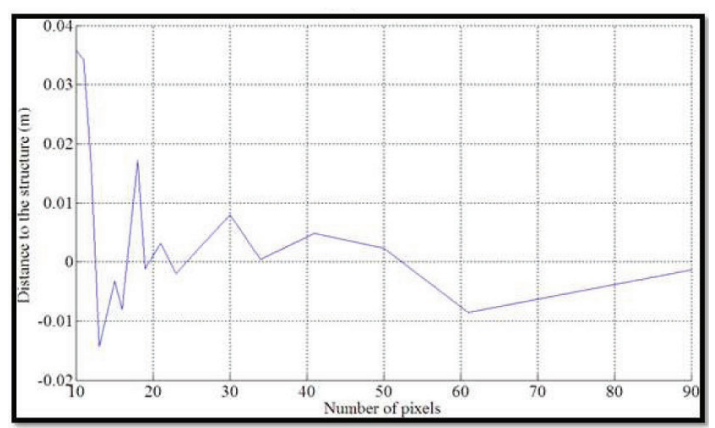

(b)

Fig. 4 Experimental calibration of the system. (a) distance to the target computed analytically and experimentally, and (b) error obtained by the Eq. 2 .

\section{Experimental results}

The results are obtained using the ROVFURG-II, developed at FURG (Universidade Federal do Rio Grande). Detailed information about the design, development and construction of this vehicle can be found in Centeno (2007) and Kuhn (2011). This vehicle has four thrusters, two arranged horizontally and two vertically, as shown in Fig. 5, where are shown the orthogonal and perspective simplified views. Currently, the ROV is equipped with camera, pressure sensor, altimeter, gyroscopes and accelerometers. 
The camera is a model Typhoon (Tritech, 2012) and does not have any kind of movement relative to the robot, being fixed to its metal frame and facing forward, i.e., the images captured are those that are in front of the vehicle. It has a resolution of $640 \times 480$ pixels, but in this paper, the images were processed at a resolution of $320 \times 240$ pixels, so that the processing time is smaller, allowing its application in real time, without loss in performance. The frame rate was fixed to $10 \mathrm{~Hz}$ due the capacities of the ROV to processing this information.

The target is the same that was used for the development and adjust the image processing algorithm, i.e., a metallic pipe-like target with $3.2 \mathrm{~cm}$ external diameter. Thus, Eq. 2 was used to obtain the distance from this structure to the vehicle. The experiment presented was repeated five times, producing similar results.

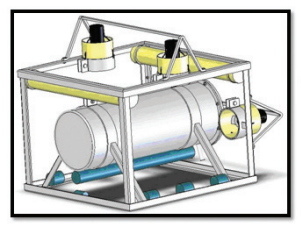

(a)

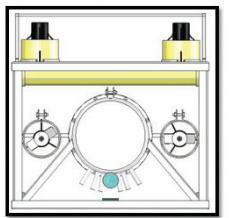

(c)

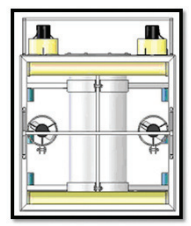

(b)

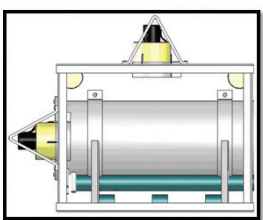

(d)
Fig. 5 Different views from the vehicle ROVFURG-II generated using CAD Software. (a) Perspective, (b) Top, (c) Frontal, and (d) Lateral view.

\subsection{Tracking a pipe}

Fig. 6 presents a sequence of images captured by video camera and processed by the proposed method. The presented frames are spaced by a second, the first refers to the instant $0 \mathrm{~s}$ and the last is related to $69 \mathrm{~s}$. In this experiment, the target was not correctly positioned vertically, so it presents an inclination degree in images. In the top left corner in each image shows the width of the target in pixels. It is converted in distance from the structure using the Eq. 2. In the center of the frame is shown the number of the images acquired from the beginning of the acquisition process. Finally, in the top right appears the instantaneous frame rate in fps (frame per second).

At certain times, the algorithm cannot identify the target. When it happens, the width information is not updated, being last valid value identified. Typically, these fails in the detection process happen by blurring process in camera acquisition, due the movement of the target. In this experiment, was during $70 \mathrm{~s}$, then, a total of 700 images were captured and processed. Of this total, the proposed method failed in identifies the target only in 48 frames, i.e., the success rate was $93.1 \%$. Moreover, there were only five consecutive failures of the structure identification, which generates a maximum delay of $500 \mathrm{~ms}$ in response, but not enough to compromise the system performance due to its slow dynamic of the process.

\subsection{Controlling a robot using the tracked target}

It was shown in the previous sections how to extract target distance information from the image analysis. This distance information is used to control the surge motion of the vehicle, which has control algorithm defined from a PID (Proportional, Integral and Derivative). In this controller, it was used a forgetting window in the integral component (Gomes and Bier, 1998). This window consists of evaluating only $\mathrm{N}$ elements to compose the sum of integral component errors. The discrete mathematical expression implemented for this controller is shown in Eq. 3.

$$
\begin{aligned}
& u_{s}\left(t_{k}\right)=K p_{s} e_{s}\left(t_{k}\right)+K i_{s} T \sum_{i=k+1-N_{s}}^{k} e_{s}\left(t_{i}\right) \\
& +K d_{s}\left(e_{s}\left(t_{k}\right)-e_{s}\left(t_{k-1}\right)\right) / T,
\end{aligned}
$$

where: $u_{s}\left(t_{k}\right)$ is the output signal of the surge motion controller in the actual instant, $K p_{s}, K i_{s}$, and $K d_{s}$ are the proportional, integral, and derivative gains, respectively, of the surge motion controller, $e_{s}\left(t_{k}\right)$ is the distance to the structure error signal sampled in the actual instant, $N_{s}$ is the number of elements in the forgetting window of the surge motion controller, $\sum_{i=k+1-N_{s}}^{k} e_{s}\left(t_{i}\right)$ is the sum of the last $N_{s}$ samples of the distance error signal, $e_{s}\left(t_{k-1}\right)$ is the distance error signal in the previous sampling instant, and $T$ is the sampling time.

The distance controlling, i.e., the surge motion controller was evaluated only using a constant distance reference. During inspect tasks, it is important to maintain the same distance to the target in order to improve the capabilities of the inspector to find possible fails in the structure. The experimental results were obtained using a trial-and-error controller tuning where the constants are: $K p_{s}=3000 ; K i_{s}=600 ; K d_{s}=60 ; N_{s}=10$ elements.

Considering a reference distance between the target and the vehicle of $0.3 \mathrm{~m}$, the target structure was positioned in front of the camera at $0.16 \mathrm{~m}$ before the controller be started. It results in an initial error of $0.14 m$. Fig. 7 shows the response of the surge motion of the system in this case. Fig. 8. shows the error signal and Fig. 9 shows the thrust of the only one frontal actuator. The vehicle is composed by two frontal actuators, but as we are interested in evaluate the surge motion controller, the signal applied in both is the same. The total thrust generated by the vehicle is the double of the shown in Fig. 9. 
Controlling a system for underwater visual inspection

Paulo L. J. Drews-Jr., Vinícius N. Kuhn and Sebastião C. P. Gomes

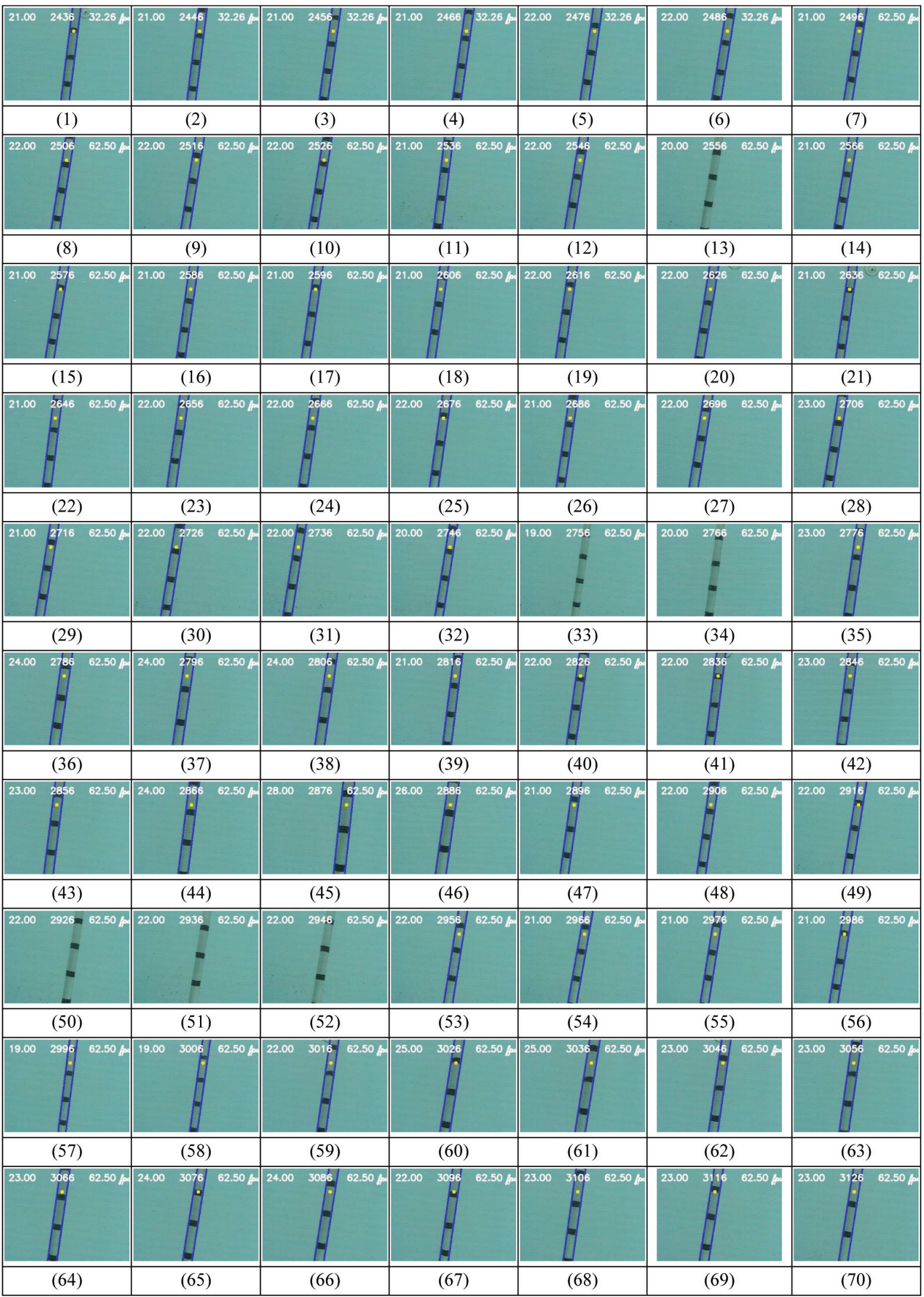

Fig. 6 Images processed by the proposed method, where the blue lines represent the cable/pipe detection: the results obtained in each second is shown, i.e., the image (1) was acquired in the start (0s), and the image (70) was acquired after $69 \mathrm{~s}$. 


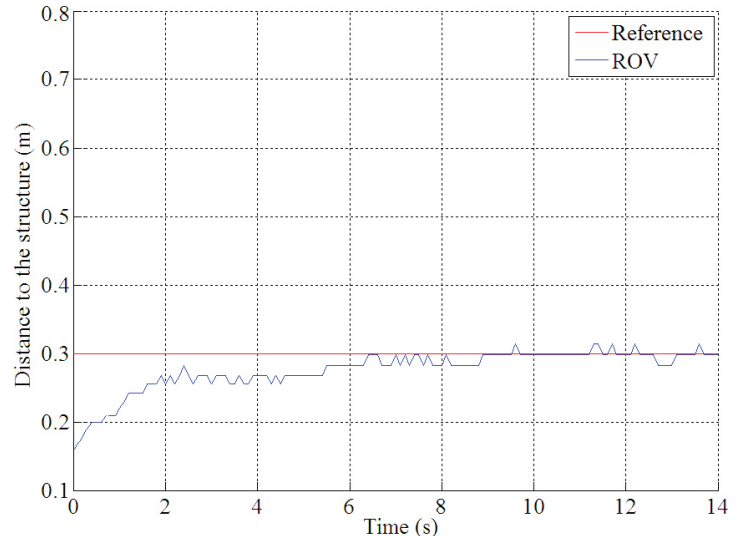

Fig. 7. Distance to the target structure during the surge motion control.

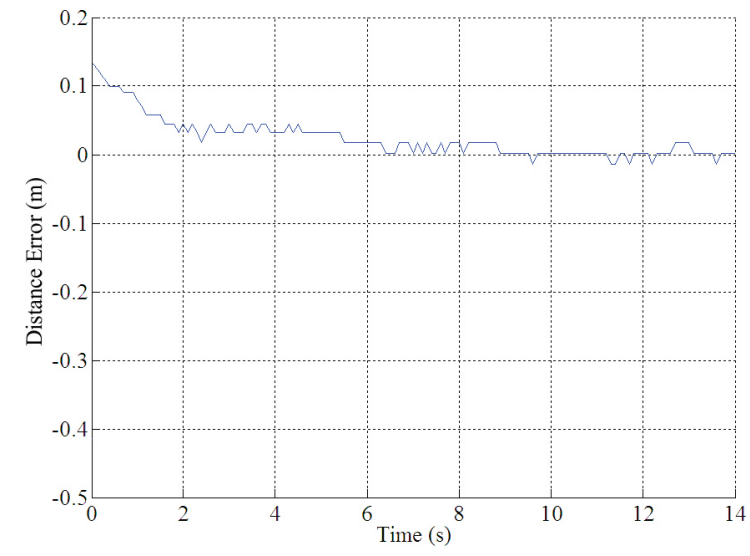

Fig. 8. Error distance between the reference and the vehicle position, estimated using the computer vision method.

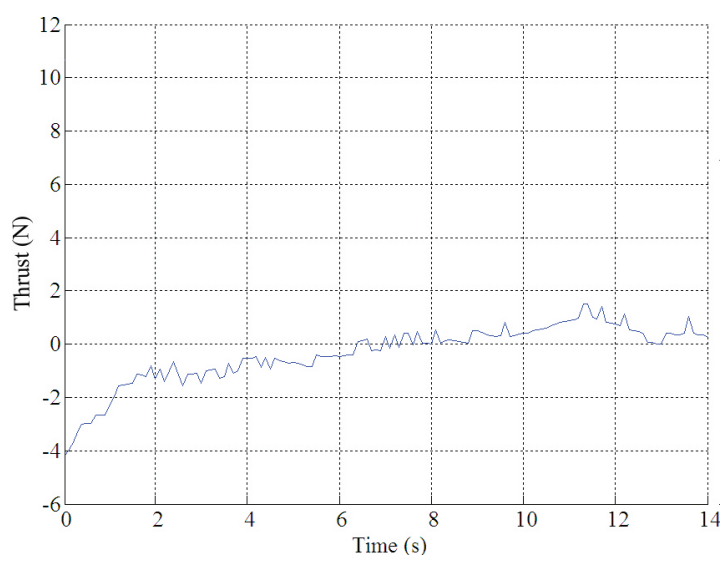

Fig. 9. Thrust of the frontal actuator during the surge motion control.

Fig. 7 shows the distance between the reference and the actual position of the vehicle. It is possible to see the fast convergence to the reference position. Fig. 8 shows that, after $1.5 \mathrm{~s}$ from the beginning, the vehicle presents a distance error smaller than $5 \mathrm{~cm}$, and after $5.5 \mathrm{~s}$, the error is smaller than $2 \mathrm{~cm}$. This steady state error could be reduced using a higher resolution in the images from camera, but it implies in a bigger time processing and the performance of the system can be compromised. Other possibility is increase the integral constant used in the system but it can compromise the stability of the system.

Other experiment was accomplished in order to test the robustness of the proposed system in disturb conditions. The target was initially set at $0.37 \mathrm{~m}$ away from the ROV and the control should keep this distance constant. Fig. 10 shows the error obtained in trying to maintain that position control constant. In order to verify the performance of the control law, manual disturbances were generated in the target structure. The target was externally moved further from the robot at the instants ' $a$ ' and ' $c$ ', in order to simulate disturb. It was moved closer at the instant ' $b$ '. As can be seen in Figure 10, the control performance was good. The error grows up after the disturbances at the instants $\mathrm{a}, \mathrm{b}$ and $\mathrm{c}$, but the control acts and does fall the error below of $0.05 \mathrm{~m}$.

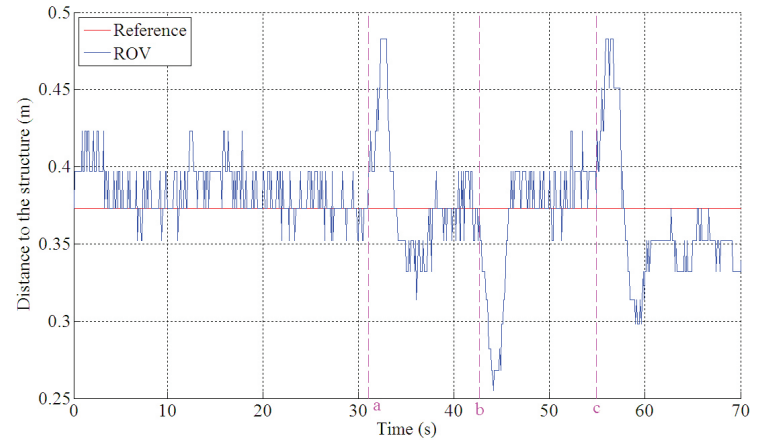

Fig. 10. Experimental result showing the error in maintaining a constant distance from the target.

\section{Conclusions}

The present paper described a method to detect and tracking cable-pipeline structure using an efficient algorithm and a PID controller. It able the method to run embedded in the vehicle with real-time performance. Results obtained using a real ROV and a metallic cable-like target was performed showing the capabilities of the proposed algorithm. Although, the results were obtained in $10 \mathrm{~Hz}$, the method is capable to run in $60 \mathrm{~Hz}$. This value is limited by the performance of the control system.

Graphically, it was observed that the results obtained had small errors in the tracking of the reference trajectory, but that in general, were visually imperceptible and are considered satisfactory for the inspection operations with ROVFURG-II. However, it is quite probable that a greater refinement of the controller parameters could further improve system performance.

An important limitation of the proposed method is the previous knowledge of the target width. In the future, we intend to develop an automatic method for online calibrate it. 
Future works will involve to implement a full controller in order to maintain a fixed distance between the ROV in all degree of freedom. Related to the computer vision method, the future directions will be focused on dealing with water turbidity and distortions since an improvement in image quality is necessary. It will able us to test this method in a real oceanic experiments.

\section{References}

CAnNy, J. (1986) - "A computational approach to edge detection". IEEE Transactions on Pattern Analysis and Machine Intelligence, vol. 8, no. 6, pp. 679-698.

Centeno, M. (2007) - "ROVFURG-II: Projeto e construção de um veículo subaquático não tripulado de baixo custo (In Portuguese)". Master's thesis, Universidade Federal do Rio Grande (FURG).

DudA, R. O. and Hart, P. E. (1972) - "Use of the hough transformation to detect lines and curves in pictures". Comm. ACM, vol. 15, pp. 11-15.

Doucet, A., Godsill, S. and Andrieu, C. (2000) - "On sequential Monte Carlo sampling methods for Bayesian filtering". Statistics and Computing, vol. 10, no. 3, pp. 197-208.

Drews JR., P. L. J., Botelho, S. S. C., Gomes, S. C. P. (2008) - "SLAM in Underwater Environment using SIFT and Topologic Maps". $5^{\text {th }}$ Latin American Robotics Symposium - LARS, Salvador, Brazil, pp. 91-96.

Drews Jr., P. L. J., Kuhn,V. N. and Gomes, S. C. P. (2012) - "Tracking System for Underwater Inspection Using Computer Vision". International Conference on Offshore and Marine Technology: Science and Innovation (NAVTEC), Rio Grande, Brazil, pp. 27-30.
Drews JR., P. L. J., Longui, J. P. C., Rosa, V. S. (2013) - "Realtime Depth Estimation for Underwater Inspection Using Dual Laser and Camera". Symposia on Automation and Computation for Naval, Offshore and Subsea Industry NAVCOMP, Rio Grande, Brazil.

Gomes, S. C. P. and Bier, C. C. (1998) - "Estudo sobre trajetórias de controle para robôs manipuladores" (In Portuguese). $12^{\text {th }}$ Congresso Brasileiro de Automática CBA, Uberlândia, Brazil.

Gomes, S. C. P., Moraes, C. E. M., Drews Jr., P. L. J., Moreira, T. G. and Tavares, A. M. (2005) - "Underwater Vehicle Dynamic Modeling". 18 $8^{\text {th }}$ International Congress of Mechanical Engineering - COBEM, Ouro Preto, Brazil, pp. 1-8.

KaLman, R. (1960) - "A new approach to linear filtering and prediction problems". Journal of Basic Engineering, vol. 82 , no. 1 , pp. 35-45.

Kunn, V. (2011) - "Controle automático de um veiculo de inspeção subaquática utilizando sensoriamento de baixo custo" (In Portuguese). Master's thesis, Universidade Federal do Rio Grande (FURG), Rio Grande, Brazil.

Kunn, V., Drews Jr., P. L. J., Gomes, S. C. P., Cunha, M. A. B., Botelho, S. S. C. (2013) - "Automatic Control of a ROV for Inspection of Underwater Structures Using a Low Cost Sensing". Accepted in Journal of the Brazilian Society of Mechanical Sciences and Engineering.

NARIMANI, M., Nazem,S. and Loueipour, M. (2009) - "Robotics vision-based system for an underwater pipeline and cable tracker". IEEE OCEANS - EUROPE, pp. 1-6.

Szeliski, R. (2010) - "Computer Vision: Algorithms and Applications”. Springer.

Tritech International Limited (2012) - “Typhoon colour zoom camera," Available online.

WIRTH,S. (2007) - "Visual underwater cable/pipeline tracking". Master's thesis, Universitat Koblenz-Landau, Germany.

ZHANG, Z. (2000) - "A flexible new technique for camera calibration". IEEE Transactions on Pattern Analysis and Machine Intelligence, vol. 22, no. 11, pp. 1330-1334. 